\title{
Interference of light from independent sources
}

\author{
David T. Pegg* \\ Centre for Quantum Computer Technology, School of Science, \\ Griffith University, Nathan, Brisbane 4111, Australia
}

(Dated: November 13, 2006)

\begin{abstract}
We extend and generalize previous work on the interference of light from independent cavities that began with the suggestion of Pfleegor and Mandel [Phys. Rev. 159, 1084 (1967)] that their observed interference of laser beams should not be associated too closely with particular states of the beams but more with the detection process itself. In particular we examine how the detection of interference induces a non-random phase difference between internal cavity states with initial random phases for a much broader range of such states than has previously been considered. We find that a subsequent interference measurement should give results consistent with the induced phase difference. The inclusion of more cavities in the interference measurements enables the construction in principle of a laboratory in the sense used by Aharonov and Susskind, made up of cavity fields that can serve as frames of phase reference. We also show reasonably simply how intrinsic phase coherence of a beam of light leaking from a single cavity arises for any internal cavity state, even a photon number state. Although the work presented here may have some implications for the current controversy over whether or not a typical laboratory laser produces a coherent state, it is not the purpose of this paper to enter this controversy; rather it is to examine the interesting quantum physics that arises for cavities with more general internal states.
\end{abstract}

PACS numbers: 03.65.Ta, 42.50.-p, 42.50.Dv

${ }^{*}$ D.Pegg@griffith.edu .au 


\section{INTRODUCTION}

Spatial and temporal interference effects for light are readily understood classically in terms of its wave nature. In terms of quantum optics, interference is a far more interesting phenomenon in relation to the oft-quoted statement of Dirac [1] that each photon interferes only with itself, with interference between different photons never occurring. Experiments on interference at the beginning of the modern quantum optics era include those of Forester et al. [2], Hanbury Brown and Twiss [3], Magyar and Mandel [4], Pfleegor and Mandel [5, 6] and subsequently by Kuo et al. [7]. Recently a renewal of interest in the interference of light from different sources has been generated by the controversy over whether or not a typical laboratory single-mode laser really produces a coherent state of light as is commonly accepted [8-11]. The experiments of Pfleegor and Mandel [5, 6] that demonstrate interference between independent lasers can be easily explained if lasers do produce coherent states and are thus sometimes considered to be evidence that this is the case (see, for example, Ref. [12] for a discussion of this). Because of this prevailing opinion, Mølmer [8], in proposing that optical coherence in laser light is merely a convenient fiction, demonstrated how two cavities containing light in equal photon number states could still exhibit interference in a homodyne experiment.

Although their optical interference experiments have been taken as evidence of optical coherence in laser light, it is interesting to note that Pfleegor and Mandel [6] actually caution against associating their observed effect too closely with particular states of the two laser beams. They advocate instead that it is better to associate the interference with the detection process itself and state that similar results should be obtainable with a wide range of possible states of the two fields being superposed. They show that the interference effect of light from the two sources can be thought of as being associated with the detection of each photon. If there is a non-zero amplitude for a photon detection event to be caused by either source, then the detection of the first photon changes the combined field state in such a way that the expression for the probability of absorption for the next photon contains interference terms. A later interference experiment involving two Fock states by Ghosh and Mandel [13] has been interpreted as follows [14]. Although the phase difference between two single-photon fields is random, and thus no classical type of optical interference is expected, the detection of the first photon reduces the state of the two fields to a simple entangled 
state that does have a reasonably well-defined phase difference and which gives the observed interference effect [14]. Later Javanainen and Yoo [15] also looked at the interference for two Fock states, but in the context of Bose-Einstein condensates, in a similar way. The issue was revived and described again in similar terms in the optical context by Mølmer [8] specifically to show that states such as Fock states lead to balanced homodyne signals that look like those from coherent states (see also Ref. [16]).

Although the controversy over whether or not a laser cavity field is actually in a coherent state has revived interest in measurement-induced localization effects associated with entanglement, it is not the intention of this paper to enter the controversy. Rather we are interested in the physics that arises when the cavity field is not assumed to be in a coherent state but, for example, is considered instead to be in an incoherent mixture of photon number states. Although these can be mathematically equivalent descriptions, the physics that arises from the latter can be more interesting. This phenomenon of a measurement-induced phase difference between two independent lasers is of particular interest as it represents a quantum effect in which the quantity that is being measured is actually created by the measurement itself. Other related work can be found in Refs. [17, 18]. In [17] cavities with identical fields have been the investigated and in [18] we investigated more general field states but only in terms of the results of the detection of a few photons. Here we generalise these investigations to include quite general optical field states with the phase difference generated by a large number of photon detection events. We find that detection-induced interference should indeed be obtainable with a wide range of possible states of the two fields being superposed in agreement with the postulate of Pfleegor and Mandel [6]. We then extend the work to include three cavities and also look at intrinsic phase coherence in a single beam of light generated by a cavity field in any state, even a photon number state. As opposed to optical coherences, that is off-diagonal elements of the density matrix in the number state representation, by phase coherence we mean a non-random phase difference between different packets of light in the beam.

\section{EFFECT OF PHOTOCOUNTING}

In this section we outline briefly the essential physics that underlies the effects that the detection and non-detection of a photon in the fields leaking from laser cavities have on the 
internal cavity fields.

\section{A. Single cavity}

When a perfect photodetector, which detects all incident photons, is used to monitor the light leaking through the partially transmitting end mirror of a laser cavity $a$, we might expect for a suitable intensity a series of photon counts separated by periods with no counts. Then the effect of the detection on the initial single-mode field state $\hat{\rho}_{a}$ inside the cavity will depend on how this state is altered by the detection of a single photon and also by a period in which no counts are observed. We assume that the external field in the continuation of the internal mode outside the cavity is initially in the vacuum state $|0\rangle_{0_{0}}\langle 0|$. Suppose some light escapes from the cavity for a very short time $\delta t$ and a measurement is made on the external field after this time. For $\delta t$ sufficiently short only one-photon and zero-photon detection events will be important. During $\delta t$ the combined internal and external state evolves unitarily to $\hat{U}|0\rangle_{\circ} \hat{\rho}_{a}\langle 0| \hat{U}^{\dagger}$ where $\hat{U}$ is the time shift operator for this short time. This state will in general be entangled. The measurement of one photon outside the cavity at the end of this interval $\delta t$ projects this state onto $|1\rangle_{0}\langle\langle 1|$ resulting in the internal state after the detection being collapsed to ${ }_{o}\langle 1|\hat{U}| 0\rangle_{\circ} \hat{\rho}_{a}\left\langle 0\left|\hat{U}^{\dagger}\right| 1\right\rangle_{0}$. This non-unitary change effectively resets the initial conditions with the collapsed state being the new internal state and the external state being returned to the vacuum by the absorption of the photon. The operator, which here is $\alpha\langle 1|\hat{U}| 0\rangle_{\circ}$, whose action gives the (unnormalized) state after the collapse is sometimes referred to as the Kraus operator [19]. For an interaction Hamiltonian containing energy-conserving terms in $\hat{a} \hat{a}_{o}^{\dagger}$ and $\hat{a}^{\dagger} \hat{a}_{o}$ where $\hat{a} \hat{a}_{0}^{\dagger}$ is the product of the internal photon annihilation operator and external photon creation operator, ${ }_{o}\langle 1|\hat{U}| 0\rangle_{\circ}$ for the short time $\delta t$ is just proportional to $\hat{a}$, and thus the internal state is changed to $\hat{a} \hat{\rho}_{a} \hat{a}^{\dagger}$ by the detection of one photon externally at the end of this time. We can write $\hat{a} \hat{\rho}_{a} \hat{a}^{\dagger}$ as $\hat{J} \hat{\rho}_{a}$ where $\hat{J}$ is a superoperator [20]. The description of a zero-photon detection event will involve the operator ${ }_{o}\langle 0|\hat{U}| 0\rangle_{\circ}$. By expanding ${ }_{o}\langle 0|\hat{U}| 0\rangle_{\circ}$ to second order, that is to include the term containing the square of the Hamiltonian, we find two ways in which the zero-photon detection event can be achieved. The first way is by means of the action of the unit operator and the second involves the action of the superoperator

$$
\widehat{L}_{a} \hat{\rho}_{a} \equiv-\frac{1}{2}\left(\hat{a}^{\dagger} \hat{a} \hat{\rho}_{a}+\hat{\rho}_{a} \hat{a}^{\dagger} \hat{a}\right)
$$


arising from the term in $\hat{a}^{\dagger} \hat{a}_{o} \hat{a} \hat{a}_{o}^{\dagger}$ acting on the initial internal state and the external vacuum. After each one or zero photon detection event the external state is returned to the vacuum, so the unconditioned evolution of the internal state should be the same as that for a cavity mode coupled to a zero temperature environment. The master equation for the latter is [20]

$$
\frac{d \hat{\rho}_{a}(t)}{d t}=2 \Gamma\left(\hat{J}+\hat{L}_{a}\right) \hat{\rho}_{a}(t)
$$

where $\Gamma$ is the cavity decay constant. Removing $\widehat{J} \hat{\rho}_{a}(t)$ from $(2)$ gives the conditioned evolution equation for a period in which no counts are observed, which has the formal unnormalized solution

$$
\begin{aligned}
\hat{\rho}_{a}(t) & \propto \exp \left(2 \Gamma t \hat{L}_{a}\right) \hat{\rho}_{a}(0) \\
& =\exp \left(-\Gamma t \hat{a}^{\dagger} \hat{a}\right) \hat{\rho}_{a}(0) \exp \left(-\Gamma t \hat{a}^{\dagger} \hat{a}\right) .
\end{aligned}
$$

A series of photon counts with intervening periods $t_{1}, t_{2}, \ldots, t_{M}$ that contain no counts will thus change the initial density operator $\hat{\rho}_{a}$ to become proportional to

$$
\widehat{J} \exp \left(2 \Gamma \widehat{L}_{a} t_{M}\right) \ldots \widehat{J} \exp \left(2 \Gamma \widehat{L}_{a} t_{2}\right) \widehat{J} \exp \left(2 \Gamma \widehat{L}_{a} t_{1}\right) \hat{\rho}_{a}
$$

(see also Refs. [20,21]). If the initial internal state is a coherent state $|\alpha\rangle$, it is straightforward to show from (4) that the final state is also a coherent state but with the mean photon number changed from $|\alpha|^{2}$ to $|\alpha|^{2} \exp (-2 \Gamma t)$, where $t=t_{1}+t_{2}+\ldots$

In addition to suffering a change due to the external detection, the state of the laser cavity will also be changed by the pumping mechanism. In this paper, where we are primarily interested in the former change, we shall assume that the total detection time is significantly less than the characteristic pumping time.

\section{B. Two cavities}

We now allow the light from cavity $a$ to interact with light of the same frequency from another laser cavity $b$ in state $\hat{\rho}_{b}$ by means of a balanced homodyne arrangement. The fields leaking from the two cavities enter the input ports of a 50:50 beam splitter with perfect photodetectors in its output ports as shown in Fig. 1. We label detector 1 as the detector that detects light transmitted through the beam splitter from cavity $a$ and light reflected by the beam splitter from cavity $b$. The other is detector 2. As shown by Mølmer 
[8], interference effects can be observed even when the cavity fields are in identical photon number states. Essentially with the light leakage the cavity fields become entangled with the external fields. Measurement of the external fields then collapses the cavity fields into a new state, which affects subsequent photodetection probabilities. For convenience, we shall assume throughout this work that both cavities, and also the third cavity that is introduced later, have identical constructions but the internal field states can be quite different. It is not difficult to generalise to cavities of different construction but we shall not do so here. The details of the effect on general field states of each photon detected for this case have been given explicitly in Ref. [18]. The detection of the first photon, in detector 1 say, alters the combined cavity state to

$$
\hat{\rho}_{1} \propto \hat{J}_{+} \hat{\rho}_{a} \hat{\rho}_{b}
$$

where

$$
\hat{J}_{+} \hat{\rho}=[\hat{a}+\exp (i \gamma) \hat{b}] \hat{\rho}\left[\hat{a}^{\dagger}+\exp (-i \gamma) \hat{b}^{\dagger}\right]
$$

where $\hat{a}$ and $\hat{b}$ are photon annihilation operators for cavities $a$ and $b$ and $\gamma$ depends on optical path lengths. For example, for equal path lengths $\gamma=\pi / 2$. Expression (5) can be normalized by dividing by its trace. This expression has a fairly simple physical interpretation. If one photon is detected outside the cavities, one photon must be annihilated from within the cavities. Because of the beam splitter arrangement, the detected photon has an amplitude of having come from either cavity, giving an amplitude for a photon to be annihilated in either cavity, which leads to expression (5). If the first detection event were in detector 2 , we would replace $\gamma$ by $\gamma+\pi$ making the state after detection proportional to $\hat{J}_{-} \hat{\rho}_{a} \hat{\rho}_{b}$ where

$$
\widehat{J}_{-} \hat{\rho}=[\hat{a}-\exp (i \gamma) \hat{b}] \hat{\rho}\left[\hat{a}^{\dagger}-\exp (-i \gamma) \hat{b}^{\dagger}\right]
$$

When no photons are detected for a time $t$ the change is given by

$$
\hat{\rho}(t) \propto \exp \left[2 \Gamma t\left(\hat{L}_{a}+\hat{L}_{b}\right)\right] \hat{\rho}(0)
$$

where

$$
\widehat{L}_{b} \hat{\rho}=-\frac{1}{2}\left[\hat{b}^{\dagger} \hat{b} \hat{\rho}+\hat{\rho}^{\dagger} \hat{b}\right] .
$$

We can combine expressions (6), (7) and (8) to produce an expression corresponding to (4) for the single cavity case. In this paper we shall be interested only in reasonably intense cavity fields and a total measurement time $t$ small enough for the depletion effect 


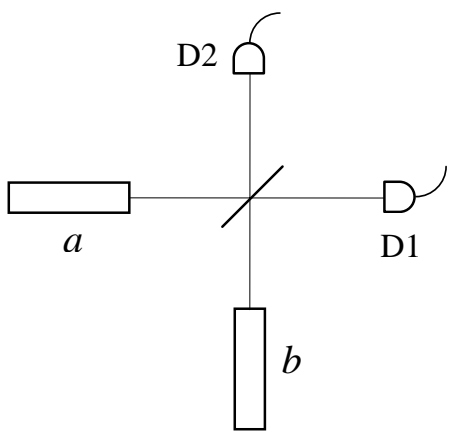

FIG. 1: Balanced homodyne arrangement. The 50:50 beam splitter transmits and reflects the light leaking from cavities $a$ and $b$ into detector 1 and detector 2 , labelled $D_{1}$ and $D_{2}$. The signal is obtained from the difference in the photocounts in the two detectors.

of the measurement to be ignored, that is for $\Gamma t<<1$. We shall assume throughout this paper that this is the case and the total measurement time is shorter than the characteristic phase diffusion time of the lasers and the characteristic pumping time. This allows us to replace $\exp \left[2 \Gamma\left(\hat{L}_{a}+\hat{L}_{b}\right) t_{m}\right]$ by the unit operator for all time intervals $t_{m}$ between onephoton measurement events. Then, after $j$ and $k$ photons are detected in detectors 1 and 2 respectively the combined (unnormalized) combined cavity state becomes

$$
\begin{aligned}
\hat{\rho}_{f} \propto & \hat{J}_{+}^{j} \hat{J}_{-}^{k} \hat{\rho}_{a} \hat{\rho}_{b} \\
= & {[\hat{a}+\exp (i \gamma) \hat{b}]^{j}[\hat{a}-\exp (i \gamma) \hat{b}]^{k} \hat{\rho}_{a} \hat{\rho}_{b} } \\
& \times\left[\hat{a}^{\dagger}-\exp (-i \gamma) \hat{b}^{\dagger}\right]^{k}\left[\hat{a}^{\dagger}+\exp (-i \gamma) \hat{b}^{\dagger}\right]^{j} .
\end{aligned}
$$

We note that if $\hat{\rho}_{a}$ is a pure coherent state, it is not affected by the measurement and likewise for $\hat{\rho}_{b}$. However if $\hat{\rho}_{a}$ and $\hat{\rho}_{b}$ are Poisson distributions of photon number states, the measurement does alter $\hat{\rho}_{a} \hat{\rho}_{b}$.

Cable et al. [17] have studied relation (10), with $\gamma=0$, for some special cases of cavity fields with identical states in each cavity. Here we shall study the more general situation in which the cavity field states need not be identical. We look firstly at states that might be expected to be found in laser cavities operating above threshold and later extend this to other possible cavity states. 


\section{LASER CAVITIES}

It is generally agreed that the best description of the field in a laser cavity is in terms of a density operator that is diagonal in the number state representation. We examine firstly a general result of this that we shall find useful later: if the initial density operators $\hat{\rho}_{a}$ and $\hat{\rho}_{b}$ are diagonal in the number state representation, then so too are the final reduced density operators $\operatorname{Tr}_{a} \hat{\rho}_{f}$ and $\operatorname{Tr}_{b} \hat{\rho}_{f}$ after the homodyning, where the traces are over the states of cavities $a$ and $b$ respectively. Thus if, after the homodyning has been completed, we perform measurements on one cavity only and not on the other then we shall not detect any optical coherences. To prove this result we note that the expansion of expression (10) will contain a large number of terms involving $\hat{a}^{m+p} \hat{\rho}_{a}\left(\hat{a}^{\dagger}\right)^{n+q} \hat{b}^{j-m+k-p} \hat{\rho}_{b}\left(\hat{b}^{\dagger}\right)^{j-n+k-q}$ where $m, n=0$, $\ldots, j$ and $p, q=0, \ldots, k$. If we find the trace over the states of cavity $b$ by using the number-state representation, we find that the contribution from all such terms will be zero except for those for which

$$
j-m+k-p=j-n+k-q
$$

because $\hat{\rho}_{b}$ is diagonal in this representation. The surviving terms will thus contain factors $\hat{J}_{a}^{m+n} \hat{\rho}_{a}$, for which all off-diagonal elements in the number state representation are zero. Thus the reduced density operator $\operatorname{Tr}_{b} \hat{\rho}_{f}$ is diagonal in this representation. Clearly a similar result holds for $\operatorname{Tr}_{a} \hat{\rho}_{f}$.

The diagonal nature of $\hat{\rho}_{a}$ also allows us to write

$$
\hat{\rho}_{a}=\sum_{n} c_{n}^{2}|n\rangle\langle n|
$$

where $c_{n}$ is positive or zero. A similar expression holds for $\hat{\rho}_{b}$. We can express (12) as

$$
\hat{\rho}_{a}=\sum_{n, n^{\prime}} c_{n} c_{n^{\prime}}|n\rangle\left\langle n^{\prime}\right| \delta_{n n^{\prime}}
$$

Using

$$
\delta_{n n^{\prime}}=(2 \pi)^{-1} \int_{0}^{2 \pi} \exp \left[i\left(n-n^{\prime}\right) \theta\right] d \theta
$$

for the Kronecker delta yields

$$
\hat{\rho}_{a}=(2 \pi)^{-1} \int_{0}^{2 \pi}|\theta\rangle_{a a}\langle\theta| d \theta
$$


where $|\theta\rangle_{a}$ is a partial phase state [22] given by

$$
|\theta\rangle_{a}=\sum_{n} c_{n} \exp (\operatorname{in} \theta)|n\rangle
$$

The mean phase of this state in a suitable $2 \pi$ phase window is $\theta$ [22]. Thus any density operator that is diagonal in the number-state representation can also represent a mixture of partial phase states with a uniform spread of mean phase. This makes it impossible, on the basis of the density operator alone, to distinguish between a Fock state of uncertain photon number and a partial phase state of unknown phase.

In this paper, as opposed to Ref. [18], we wish to study the case in which a large number $j+k$ of photocounts occur. As mentioned earlier, we do not want the cavities to be significantly depleted of photons after such an experiment so we specify that the mean photon number in each cavity is substantially in excess of this, that is, the lasers are reasonably intense.

\section{A. Different Poisson distributions}

It is useful to study the simplest case first. This is when each internal field number-state distribution is Poissonian, as is often taken to be the case for laser cavities. We shall not restrict the mean photon numbers to be equal, however, as done in previous work. In this case the partial phase states given by (16) become coherent states $|\alpha\rangle_{a}$ and we can write

$$
\hat{\rho}_{a}=(2 \pi)^{-1} \int_{0}^{2 \pi}|\alpha\rangle_{a a}\langle\alpha| d \theta
$$

where $\alpha=|\alpha| \exp (i \theta)$. We similarly write for the other cavity state

$$
\hat{\rho}_{b}=(2 \pi)^{-1} \int_{0}^{2 \pi}|\beta\rangle_{b b}\langle\beta| d \varphi
$$

with $\beta=|\beta| \exp (i \varphi)$. Then using the fact that $|\alpha\rangle$ and $|\beta\rangle$ are eigenstates of $\hat{a}$ and $\hat{b}$ allows us to write (10) eventually as

$$
\hat{\rho}_{f} \propto \int_{0}^{2 \pi} \int_{0}^{2 \pi} F(\eta, \Delta)|\alpha\rangle_{a a}\langle\alpha|| \beta\rangle_{b b}\langle\beta| d \theta d \varphi
$$

where

$$
F(\eta, \Delta)=(1+\eta \cos \Delta)^{j}(1-\eta \cos \Delta)^{k}
$$


with $\Delta=\varphi+\gamma-\theta$ and

$$
\eta=\frac{2|\alpha||\beta|}{|\alpha|^{2}+|\beta|^{2}} .
$$

We can write $|\alpha|^{2}$ and $|\beta|^{2}$ as $\bar{n}_{a}$ and $\bar{n}_{b}$ respectively, which are the initial mean photon numbers in the cavities $a$ and $b$ [20]. Then $\eta$ becomes the ratio of the geometric mean to the arithmetic mean of the mean photon numbers. By calculating $\operatorname{Tr}\left(\hat{a}^{\dagger} \hat{a} \hat{\rho}_{f}\right)$, where the right side of (19) is normalized by division by its trace, we can show that $\bar{n}_{a}$ and $\bar{n}_{b}$ are also the mean photon numbers for the final state, which is a consequence of our approximation of a negligible depletion effect.

Differentiation with respect to $\Delta$ shows that $F(\eta, \Delta)$ has equal maxima for $\Delta= \pm \Delta_{0}$ where

$$
\cos \Delta_{0}=\frac{1}{\eta} \frac{j-k}{j+k}
$$

For the symmetric case, that is $\bar{n}_{a}=\bar{n}_{b}$, we have $\eta=1$ and so the maxima occur at $\pm \Delta_{0 S}$ where $\cos \Delta_{0 S}=(j-k) /(j+k)$. It is possible in this case for all the photocounts be recorded in one detector, giving a single maximum at $\Delta=0$ or at $\Delta= \pm \pi$, which is one of the cases studied in Ref. [17]. For the asymmetric case $\eta<1$ it is very unlikely for all the photocounts to be in one detector and $F(\eta, \Delta)$ will in general have two separate peaks at $\Delta= \pm \Delta_{0}$. $F(\eta, \Delta)$ becomes more sharply peaked about these values with increasing $j$ and $k$. For the symmetric case, it is possible to show by use of an expression derived in [23] that the shape of each peak approximates a Gaussian with a width of order $(j+k)^{-1 / 2}[17]$. Consider the curve for the more general asymmetric case in the vicinity of the peak at $\Delta=\Delta_{0}$. For $\Delta-\Delta_{0}$ sufficiently small, we can write

$$
\begin{aligned}
& F(\eta, \Delta) \approx {\left[1+\cos \Delta_{0 S}-\left(\Delta-\Delta_{0}\right) \xi \sin \Delta_{0 S}\right]^{j} } \\
& \times\left[1-\cos \Delta_{0 S}+\left(\Delta-\Delta_{0}\right) \xi \sin \Delta_{0 S}\right]^{k}
\end{aligned}
$$

where $\xi=\eta \sin \Delta_{0} / \sin \Delta_{0 S}$. For $\eta=1$, the symmetric case, this reduces to

$$
\begin{aligned}
& F(1, \Delta) \approx {\left[1+\cos \Delta_{0 S}-\left(\Delta-\Delta_{0 S}\right) \sin \Delta_{0 S}\right]^{j} } \\
& \times\left[1-\cos \Delta_{0 S}+\left(\Delta-\Delta_{0 S}\right) \sin \Delta_{0 S}\right]^{k}
\end{aligned}
$$

Comparing (23) with (24) shows that if we plot $F(\eta, \Delta)$ against $\left(\Delta-\Delta_{0}\right) \xi$ we obtain the same shaped peak as we would by plotting $F(1, \Delta)$ against $\Delta-\Delta_{0 S}$. Therefore plotting $F(\eta, \Delta)$ against $\Delta-\Delta_{0}$ will give an approximate Gaussian peak with a width broadened by 
a factor $1 / \xi$ compared to the symmetric case. Thus, provided this factor stays reasonably constant, which we show below is indeed the case, the peak becomes sharper as the number of photocounts increases. The same argument applies to the peak at $\Delta=-\Delta_{0}$.

In the limit of a large number of photocounts being recorded the peaks become very sharp and the state (19) is collapsed to become

$$
\hat{\rho}_{f}=\frac{1}{2} \hat{\rho}_{f 1}+\frac{1}{2} \hat{\rho}_{f 2}
$$

where

$$
\hat{\rho}_{f 1} \propto \int_{0}^{2 \pi}|\alpha\rangle_{a a}\left\langle\alpha \| \beta^{\prime}(\theta)\right\rangle_{b b}\left\langle\beta^{\prime}(\theta)\right| d \theta
$$

with

$$
\beta^{\prime}(\theta)=|\beta| \exp \left[i\left(\theta-\gamma+\Delta_{0}\right)\right]
$$

Changing $\Delta_{0}$ to $-\Delta_{0}$ in (26) and (27) gives $\hat{\rho}_{f 2}$. The mean phase of a partial phase state in a suitably chosen $2 \pi$ window is the argument of its complex amplitude. This can be shown by use of the formalism of [22] involving an operator, which we write here as $\hat{\phi}_{a}$, acting on states of cavity $a$ fields in a sufficiently large Hilbert space. Thus the mean phase of $|\alpha\rangle_{a}$ is $\theta$. The corresponding phase variance of the coherent state $|\alpha\rangle_{a}$ is $1 /\left(4 \bar{n}_{a}\right)$ [22], so for such a state with reasonably high intensity, as we are assuming here, the phase distribution is quite sharp, allowing us to write the approximation

$$
\exp \left(-i \hat{\phi}_{a}\right)|\alpha\rangle_{a} \approx \exp (-i \theta)|\alpha\rangle_{a}
$$

From (28) and the corresponding relation for the other cavity, we find

$$
\exp \left[i\left(\hat{\phi}_{b}-\hat{\phi}_{a}\right)\right] \hat{\rho}_{f 1} \approx \exp \left[i\left(-\gamma+\Delta_{0}\right)\right] \hat{\rho}_{f 1}
$$

It follows that $\hat{\rho}_{f 1}$ is a state of reasonably well-defined phase difference, which is given by $-\gamma+\Delta_{0}$. This is despite the fact the individual phases of each cavity field are completely random, that is, there is no preferred value of phase for either cavity. This latter result follows from the integration in (26). Also, it is straightforward to show that both $\langle\hat{a}\rangle$ and $\langle\hat{b}\rangle$ are zero as expected for states of random phase. Likewise $\hat{\rho}_{f 2}$ is a state of well-defined phase difference $-\gamma-\Delta_{0}$. Thus we see that the measurement has reduced the state of the cavities to $\hat{\rho}_{f}$ for which the cosine of the sum of the phase difference and $\gamma$ has a single well-defined value, $\cos \Delta_{0}$, given by $(22)$. 
The states $|\alpha\rangle_{a}\left|\beta^{\prime}(\theta)\right\rangle_{b}$ and the corresponding state with $\Delta_{0}$ replaced by $-\Delta_{0}$ are eigenstates of $\hat{a}+\exp (i \gamma) \hat{b}$ and $\hat{a}-\exp (i \gamma) \hat{b}$, that is of the Kraus operators in (10). It is straightforward to show from this that further photocounting merely multiplies both $\hat{\rho}_{f 1}$ and $\hat{\rho}_{f 2}$ by the same factor. This does not alter (25) after renormalization and thus the state (25) is stable against further photocounting.

An interesting question arises as to what will be the outcome of a subsequent balanced homodyne measurement carried out on state (25), that is, after the initial measurement has created state (25), for example with the same apparatus used for the initial measurement. The expectation value of the number of photons detected in detector 1 in a short time interval after the initial measurement will be proportional to the probability $P_{1}$ that the first photon of the subsequent experiment is detected in detector 1. From the general formula given in [18] we find that this is given by

$$
P_{1} \propto \operatorname{Tr}\left(\hat{J}_{+} \hat{\rho}_{f}\right)
$$

where the trace is over the internal states of both cavities. The corresponding expression for the probability $P_{2}$ of detecting the photon in detector 2 is found by changing $\gamma$ to $\gamma+\pi$ [18] and is proportional to $\operatorname{Tr}\left(\hat{J}_{-} \hat{\rho}_{f}\right)$. The subsequent mean balanced homodyne signal is obtained from $P_{1}-P_{2}$, which is proportional to

$$
\operatorname{Tr}\left\{\left[\hat{a} \hat{b}^{\dagger} \exp (-i \gamma)+\hat{a}^{\dagger} \hat{b} \exp (i \gamma)\right] \hat{\rho}_{f}\right\}
$$

We find by using (25) that signal (31) is proportional to $2 \bar{n}_{a}^{1 / 2} \bar{n}_{b}^{1 / 2} \cos \Delta_{0}$. For comparative purposes, we can calculate the mean balanced homodyne signal obtained from two pure coherent states $|\alpha\rangle_{a}$ and $|\beta\rangle_{b}$ by replacing $\hat{\rho}_{f}$ in (31) by $|\alpha\rangle_{a}|\beta\rangle_{b}{ }_{b}\left\langle\left.\beta\right|_{a}\langle\alpha|\right.$. We find that this signal is proportional to $2|\alpha||\beta| \cos (\theta-\varphi-\gamma)$, where $\varphi-\theta$ is the difference in mean phases of the two coherent states. This is also the signal that we would obtain from two classical fields with phase difference $\varphi-\theta$ and field strengths $|\beta|$ and $|\alpha|$. Thus the subsequent mean balanced homodyne signal obtained from (31) is the same as that which would be obtained from two classical fields with a phase difference of $-\gamma \pm \Delta_{0}$ and field strengths proportional to $\bar{n}_{b}^{1 / 2}$ and $\bar{n}_{a}^{1 / 2}$.

Further, we find from (22) and (21) that the subsequent homodyne signal is just proportional to

$$
2 \bar{n}_{a}^{1 / 2} \bar{n}_{b}^{1 / 2} \cos \Delta_{0}=\left(\bar{n}_{a}+\bar{n}_{a}\right) \frac{(j-k)}{(j+k)}
$$


Similarly we find from (25), (30), (22) and (21) that the ratio $P_{1} / P_{2}$ of the probabilities of detecting a photon in detectors 1 and 2 for the subsequent homodyning is $j / k$, which is just the ratio of these probabilities as determined by the photocount statistics from the first homodyning. Consequently further photocounting after the initial measurement will not alter the ratio of the difference in photocounts to the total photocounts. As this determines $\cos \Delta_{0}$ and $\cos \Delta_{0 S}$, and thus $\sin \Delta_{0}$ and $\sin \Delta_{0 S}$, the factor $\xi$ therefore remains constant as the number of photocounts increases, as required above. Thus after a sufficient number of photocounts the cavity fields develop a phase difference with two possible well-defined values, even though each cavity still has a random phase. This phase difference is just that which we would infer from the homodyne measurement of two classical fields. The correlation between the signal from the first homodyne measurement and that of the subsequent measurement, provided this is done within the phase diffusion time, may be taken as indicating a phase coherence between sequential packets of light emitted by the laser cavity as discussed later.

The state (25) exhibits two values of phase difference and is stable against further homodyning with the same apparatus as that which produced the state. An interesting question concerns what will happen if we continue homodyning but with the geometry changed to give a different value of $\gamma$. Specifically, suppose we place a phase shifter in one of the input ports to alter $\gamma$ to $\gamma-\pi / 2$. In this case a photocount in either detector will no longer affect the terms $\hat{\rho}_{f 1}$ and $\hat{\rho}_{f 2}$ in (25) equally as it did when $\gamma$ was unaltered. For example, if the first photocount after the change is in detector 1 , the expression for the subsequent collapsed state is changed from $\hat{J}_{+} \hat{\rho}_{f}$ to

$$
[\hat{a}-i \exp (i \gamma) \hat{b}] \hat{\rho}_{f}\left[\hat{a}^{\dagger}+i \exp (-i \gamma) \hat{b}^{\dagger}\right]
$$

With $\hat{\rho}_{f}$ given by (25), we find that the ratio of the weightings of $\hat{\rho}_{f 1}$ and $\hat{\rho}_{f 2}$ in the collapsed state becomes $\left(1+\eta \sin \Delta_{0}\right):\left(1-\eta \sin \Delta_{0}\right)$. The ratio of the probabilities $P_{11}$ and $P_{12}$ that the second photocount is in detector 1 or detector 2 respectively can then be found to be

$$
\frac{P_{11}}{P_{12}}=\frac{1+\eta^{2} \sin ^{2} \Delta_{0}}{1-\eta^{2} \sin ^{2} \Delta_{0}} .
$$

Thus although the probability that the first photocount is in a particular detector is $50 \%$, the probability that the second photocount is in the same detector as the first is enhanced. Such an event will change the state in a way that further enhances the probability that the third photocount will be in the same detector as the first two. This leads to an instability 
that will eventually remove one of the terms $\hat{\rho}_{f 1}$ or $\hat{\rho}_{f 2}$ leaving us with a single well-defined phase difference. Whichever term remains will be unaltered by a photocount in detector 1 or detector 2, so we again have a stable equilibrium. Thus the result of further homodyning with an altered $\gamma$ is eventually to change the state to one with a single phase difference which is the same as one of the original two possible values of phase difference. That is, the original double-peaked phase difference probability distribution will collapse to a singlepeaked distribution. The two peaks have an equal chance of being the one that survives (see also Ref. [17]).

Before proceeding to the general case it is worth examining the coherent state case in a little more detail. For a single cavity the effect of detecting a single photon outside the cavity is to change the internal state to $\widehat{J}_{a} \hat{\rho}_{a}$. If the internal state is a coherent state, this state is unchanged, after renormalization, by the detection. This can be readily understood because the external field is not entangled with the internal field if this is in a coherent state [18]. Likewise if two cavity fields are prepared with a pure coherent state field in each, the homodyne measurement will have no physical effect on the internal fields, that is, $\hat{\rho}_{1}$ in $(5)$ will be the same, after renormalization, as $\hat{\rho}_{a} \hat{\rho}_{b}$. On the other hand, if the cavities were prepared in photon number states the measurement would physically alter the state because of the entanglement between the internal and external states.

Expression (12) is what we would write for the density operator if we knew that the cavity was prepared in a photon number state but did not know which one. The coefficients $c_{n}^{2}$ would then be preparation probabilities. Expression (17) is what we would write if we knew the cavity was prepared in a coherent state with a particular mean photon number but had no knowledge of its mean phase. Then $d \theta /(2 \pi)$ would be the a priori probability that the state was prepared with a mean phase between $\theta$ and $\theta+d \theta$. Mathematically (12) and (17) are identical and will thus be changed in precisely the same way by the detection of an external photon. There are different physical mechanisms, however, for the way in which this change occurs. In the coherent state case, the individual coherent states in the integrand of (17) are not changed. What is changed as we obtain information from the measurement is the probability that particular states were prepared. This information increases as more photons are detected and the a priori probabilities become a posteriori probabilities. We can regard the measurement in this case as simply giving us more information about which states in the cavities could actually have been prepared without affecting the states themselves. This 
process is called retrodiction $[24,25]$. In the number state case, the individual number states themselves are physically altered. It may seem strange that precisely the same mathematical operators, the Kraus operators, are used to find the retrodicted density operator in the coherent state case as are used to find the measurement-collapsed density operator in the number-state case. A formal proof of this is, however, given in [18]. Essentially this may be regarded as a manifestation of the preferred ensemble fallacy [9, 26, 27], which says that the

probability of the outcome of any subsequent measurement on the system is independent of how we partition the density operator. This allows us, for the purposes of predicting the statistics of the results of subsequent measurements on the laser beam and not on the atoms in the cavity, to treat the laser cavities as though they were prepared in single coherent states of definite but unknown mean phase, even if this is not the case.

\section{B. More general case}

From the preceding section we see that it is possible to find the correct mathematical expression for the density operator after the homodyne measurement by treating the cavities as though they were prepared in coherent states of known mean photon number number but unknown mean phase, provided the number-state coefficients in (12) have a Poisson distribution. This can then be treated as a simple case of retrodiction. For distributions that are not Poissonian, however, even though we can treat the cavities as being prepared in partial phase states (16) of unknown mean phase, these partial phase states can result in the external field being entangled with the internal field, in which case the measurement physically alters the state. For example, in his original work Mølmer [8] examined numerically the case of the same single photon number state in each cavity and Cable et al. [17] examined the same symmetric case analytically. Evidence of an induced phase difference was quite apparent in both approaches. As the corresponding partial phase states in this case are single photon number states, which are not even approximate eigenstates of the annihilation operator, retrodiction cannot be invoked for an explanation. It is worthwhile, therefore, to extend our approach to include more general asymmetric states. In this generalization, however, we shall still assume that the mean photon numbers of the initial cavity states are sufficiently large for depletion to be ignored after a reasonable number of photocounts. Also we still assume that $\hat{\rho}_{a}$ and $\hat{\rho}_{b}$ have no optical coherences. 
We express $\hat{\rho}_{a}$ and $\hat{\rho}_{b}$ in terms of the Glauber-Sudarshan P-representation [20] as

$$
\hat{\rho}_{a}=\int_{0}^{2 \pi} \int_{0}^{\infty}|\alpha| P(\alpha)|\alpha\rangle\langle\alpha|d| \alpha| d \theta
$$

where $P(\alpha)$ is the $\mathrm{P}$-function. We might regard (35) as representing a mixture of coherent states $|\alpha\rangle$ of uncertain mean phase and uncertain mean photon number. A corresponding expression for $\hat{\rho}_{b}$ can be written in terms of $P(\beta)$ and $|\beta\rangle$ with $\beta=|\beta| \exp (i \varphi)$. Here we are not including subscripts $a$ and $b$ as the cavity identification is reasonably clear.

Expression (10) for $\hat{\rho}_{f}$ after $j+k$ photocounts then becomes

$$
\begin{aligned}
\hat{\rho}_{f} \propto & \int_{0}^{2 \pi} \int_{0}^{\infty} \int_{0}^{2 \pi} \int_{0}^{\infty}|\alpha||\beta| P(\beta) P(\alpha) \\
& \times|r(\eta, \Delta)|^{2}|\beta\rangle\langle\beta|| \alpha\rangle\langle\alpha|d| \alpha|d \theta d| \beta| d \varphi
\end{aligned}
$$

where

$$
|r(\eta, \Delta)|^{2}=F(\eta, \Delta)\left(|\alpha|^{2}+|\beta|^{2}\right)^{j+k}
$$

with $F(\eta, \Delta)$ again given by $(20), \eta$ given by (21) and $\Delta=\varphi+\gamma-\theta$. In the general case in which we are interested, $\hat{\rho}_{a}$ and $\hat{\rho}_{b}$ have no optical coherences, that is, they are diagonal in the number-state basis. We can show from this that $P(\alpha)$ and $P(\beta)$ will be independent of $\theta$ and $\varphi$ and so can be taken outside the respective integrals. Using (37) we find that the delta-function type of behaviour of $F(\eta, \Delta)$ then yields

$$
\hat{\rho}_{f}=\frac{1}{2} \hat{\rho}_{f 1}+\frac{1}{2} \hat{\rho}_{f 2}
$$

where

$$
\begin{aligned}
\hat{\rho}_{f 1} \propto & \int_{0}^{\infty} \int_{0}^{\infty}|\alpha||\beta| P(\beta) P(\alpha)\left(|\alpha|^{2}+|\beta|^{2}\right)^{j+k} \\
& \times \int_{0}^{2 \pi}\left|\beta^{\prime}(\theta)\right\rangle\left\langle\beta^{\prime}(\theta)|| \alpha\right\rangle\langle\alpha|d \theta d| \alpha|d| \beta|
\end{aligned}
$$

with $\beta^{\prime}(\theta)$ again given by $(27)$. $\hat{\rho}_{f 2}$ is similar to (39) but with $-\Delta_{0}$ in place of $\Delta_{0}$. Because $\Delta_{0}$, as given by (21) and (22), is a function of $|\alpha|^{2}$ and $|\beta|^{2}$, the approximate relation (29) will not hold in general, even when the component coherent states have reasonably intensity.

Further photocounting after the state $\hat{\rho}_{f}$ has been established will give a subsequent mean signal that can be found from (31). Using (38), (39) and the expression for $\hat{\rho}_{f 2}$, we find that the subsequent mean signal is proportional to

$$
\begin{aligned}
2 \int_{0}^{\infty} \int_{0}^{\infty} & P(\alpha) P(\beta)\left(|\alpha|^{2}+|\beta|^{2}\right)^{j+k} \\
& \times \cos \Delta_{0}|\alpha|^{2}|\beta|^{2} d|\alpha| d|\beta| .
\end{aligned}
$$


From (21) and (22) this reduces to give the signal proportional to

$$
\begin{aligned}
& \frac{j-k}{j+k} \int_{0}^{\infty} \int_{0}^{\infty}|\beta||\alpha| P(\alpha) P(\beta) \\
& \times\left(|\alpha|^{2}+|\beta|^{2}\right)^{j+k+1} d|\alpha| d|\beta| .
\end{aligned}
$$

A corresponding calculation to find $\operatorname{Tr}\left[\left(\hat{a}^{\dagger} \hat{a}+\hat{b}^{+} \hat{b}\right) \hat{\rho}_{f}\right]$ yields, in place of (41),

$$
\int_{0}^{\infty} \int_{0}^{\infty}|\beta||\alpha| P(\alpha) P(\beta)\left(|\alpha|^{2}+|\beta|^{2}\right)^{j+k+1} d|\alpha| d|\beta|
$$

From this, the expression (31), to which the subsequent mean homodyne signal is proportional, becomes

$$
\left(\bar{n}_{a}+\bar{n}_{b}\right) \frac{j-k}{j+k}
$$

where $\bar{n}_{a}$ and $\bar{n}_{b}$ are the mean photon numbers for the state $\hat{\rho}_{f}$. This is the same our previous result (32) for fields expressible as mixtures of coherent states of known amplitude but unknown mean phase. We see, therefore, that the assumption that the fields are expressible as such mixtures is not essential in order to explain the mean signal. From Eq. (32) and the discussion preceding it, signal (43) is the same as the mean balanced homodyne signal for two pure coherent states with appropriate intensities and mean phase difference.

The fact that the mean balanced homodyne signal is non-zero and depends on $\gamma$ shows that the phase difference between the fields inside the two cavities is not random, that is, it has a non-uniform distribution. As we know the state of the cavities, we could calculate the phase distribution by the method used in [18] for calculating the phase difference distribution after the detection of the first photon. It is simpler and more convenient here, however, where the fields of interest are strong, to investigate the phase relationship of the two fields by initially working in terms of an operator defined by

$$
\hat{C}(\gamma)=\hat{a} \hat{b}^{\dagger} \exp (-i \gamma)+\hat{a}^{\dagger} \hat{b} \exp (i \gamma)
$$

Although this operator acts on the internal states of the cavities, we see from (31) that the expectation value of this operator is proportional to the homodyne signal of the external fields. As we shall see below, an interesting property of the two internal fields can be ascertained by finding the variance of $\hat{C}(\gamma)$ for $\hat{\rho}_{f}$. This is simplest to do firstly for the case in which both fields have initial Poisson distributions. The two important factors involved in determining the variance of $\hat{C}(\gamma)$ are the width of the function $F(\eta, \Delta)$ in Eq. (20) and 
the widths of the Poisson distributions. If we assume, as we have been doing, that the total number of photocounts is large enough to give $F(\eta, \Delta)$ a delta-function type of behaviour then it is not difficult to show that the variance of $\hat{C}(\gamma)$ is $\bar{n}_{a}+\bar{n}_{b}$, which is just the sum of the variances of the two Poisson distributions. In order to avoid depletion effects, our assumption has been that $j+k$ is much less than the mean number of photons in each cavity. This means that in order to treat $F(\eta, \Delta)$ like a delta function, the fields themselves must be quite intense, which implies that $\left(\bar{n}_{a}+\bar{n}_{b}\right)^{1 / 2}$ must be negligible relative to the mean photon numbers. Thus the root of the variance of $\hat{C}(\gamma)$ will also be relatively small.

Calculating the variance of $\hat{C}(\gamma)$ for the more general case is more complicated. However, by using the same assumption as above to ignore terms of order $\bar{n}_{a}$ and $\bar{n}_{b}$ in comparison to terms of order $\bar{n}_{a}^{2}, \bar{n}_{b}^{2}$ and $\bar{n}_{a} \bar{n}_{b}$, we eventually find, by using method similar to that which we used to calculate the mean signal, the variance of $\hat{C}(\gamma)$ to be

$$
\begin{aligned}
\Delta C^{2}= & \left(\overline{n_{a}^{2}}+\overline{n_{b}^{2}}+2 \overline{n_{a} n_{b}}-\bar{n}_{a}^{2}-\bar{n}_{b}^{2}-2 \bar{n}_{a} \bar{n}_{b}\right) \\
& \times \frac{(j-k)^{2}}{(j+k)^{2}} \\
\approx & \left(\Delta n_{a}^{2}+\Delta n_{b}^{2}\right) \frac{(j-k)^{2}}{(j+k)^{2}} .
\end{aligned}
$$

Approximating $\overline{n_{a} n_{b}}$ by $\bar{n}_{a} \bar{n}_{b}$ in (45) is justified within the negligible depletion assumption above, which essentially allows us to ignore the effect on $\overline{n_{a} n_{b}}$ of any entanglement that will in general arise from the detection of the $j+k$ photons. Thus the root of $\Delta C^{2}$ for non-Poisson photon number distributions will also be relatively small provided these distributions are relatively narrow.

The smallness of $\Delta C^{2}$ allows us to write the approximation

$$
\hat{C}(\gamma) \hat{\rho}_{f} \approx\langle\hat{C}(\gamma)\rangle \hat{\rho}_{f}
$$

We can understand the significance of this by considering, for simplicity, the case in which $\gamma=0$ so $\hat{C}(\gamma)$ is just $\hat{a} \hat{b}^{\dagger}+\hat{a}^{\dagger} \hat{b}$. We can use the formalism of Ref. [22] to show that when this operator acts on physical states, it can be written as

$$
\begin{aligned}
\hat{C}(0)= & \exp \left[i\left(\hat{\phi}_{a}-\hat{\phi}_{b}\right)\right] \hat{n}_{a}^{1 / 2}\left(\hat{n}_{b}+1\right)^{1 / 2} \\
& +\exp \left[-i\left(\hat{\phi}_{a}-\hat{\phi}_{b}\right)\right] \hat{n}_{b}^{1 / 2}\left(\hat{n}_{a}+1\right)^{1 / 2}
\end{aligned}
$$

where $\exp \left[i\left(\hat{\phi}_{a}-\hat{\phi}_{b}\right)\right]$ is the operator for the phase difference of the two cavity fields. For 
intense fields with narrow number state distributions, we obtain from (47)

$$
\hat{C}(0) \hat{\rho}_{f} \approx 2 \cos \left(\hat{\phi}_{a}-\hat{\phi}_{b}\right) \bar{n}_{a}^{1 / 2} \bar{n}_{b}^{1 / 2} \hat{\rho}_{f}
$$

From (47) and (48) we see, using the fact that $\langle\hat{C}(0)\rangle$ is given by (43), that

$$
\begin{aligned}
\cos \left(\hat{\phi}_{a}-\hat{\phi}_{b}\right) \hat{\rho}_{f} & \approx \frac{\langle\hat{C}(0)\rangle}{2 \bar{n}_{a}^{1 / 2} \bar{n}_{b}^{1 / 2}} \hat{\rho}_{f} \\
& =\frac{\left(\bar{n}_{a}+\bar{n}_{b}\right)(j-k)}{2 \bar{n}_{a}^{1 / 2} \bar{n}_{b}^{1 / 2}(j+k)} \hat{\rho}_{f}
\end{aligned}
$$

Thus the cavity fields have a sharply defined cosine of phase difference given by

$$
\cos \bar{\Delta}_{0}=\frac{1}{\bar{\eta}} \frac{j-k}{j+k} .
$$

where

$$
\bar{\eta}=\frac{2 \bar{n}_{a}^{1 / 2} \bar{n}_{b}^{1 / 2}}{\bar{n}_{a}+\bar{n}_{b}} .
$$

When $\gamma \neq 0, \cos \bar{\Delta}_{0}$ is the well-defined value of the cosine of the sum of the phase difference and $\gamma$. This result applies to all intense cavity fields with initial density matrices that are diagonal in the photon number state basis, provided the photon number probability distributions are reasonably narrow. This is consistent with Ref. [18] in which we found that the interference effects depended on the narrowness of the number state distributions.

\section{THIRD CAVITY}

We now introduce a third cavity $c$ whose field state also has no optical coherences. Before any measurements are made, the phase difference between the field in this cavity and the field in cavity $a$ will be random. We look firstly at how the alteration of the states of cavities $a$ and $b$ to produce a well-defined cosine of the sum of the phase difference and $\gamma$ affects the random relative phase between cavity $a$ and cavity $c$. To be definite, let the combined state of cavities $a$ and $b$ be $\hat{\rho}_{f}$ given by (10). For Poisson number-state distributions in the cavities $\hat{\rho}_{f}$ is given by (25) and (26) but for more general states it will be a more complicated expression following from (36). We write $\bar{\rho}_{f}=\operatorname{Tr}_{b} \hat{\rho}_{f}$ for the reduced density operator for cavity $a$ and let the initial state of cavity $c$ be $\hat{\rho}_{c}$. Because the density matrix of $\bar{\rho}_{f}$ in the number state representation is also diagonal, as we showed earlier, the phase difference between the fields of cavities $a$ and $c$ will be random. The diagonal nature of the density 
matrix for the field in cavity $a$ is the same initial condition that we have used for our previous calculations. Thus the eventual signal obtained from a balanced homodyne measurement of the $a$ and $c$ fields will be independent of whether or not a balanced homodyne measurement of the $a$ and $b$ fields is done first, apart from a small change in $\bar{n}_{a}$ due to some depletion, which we assuming here to be negligible.

The next question we ask is as follows. Suppose we make a homodyne measurement of the fields of cavities $a$ and $b$ for a sufficient time to establish a reasonable phase difference. We have seen that an immediately subsequent homodyne measurement would yield a result in accord with this difference. Suppose also, however, that after the first homodyne measurement of the $a$ and $b$ fields, we use another homodyne measurement to establish a phase difference between cavity fields $a$ and $c$ and only then perform the subsequent measurement of the $a$ and $b$ fields. Will this yield the same result as an immediately subsequent measurement would? That is, will the intervening measurement of the $a$ and $c$ fields upset the phase relationship between $a$ and $b$ ? As before, after the first measurement of the $a$ and $b$ fields the unnormalized state will be of the form $\hat{f} \hat{\rho}_{a} \hat{\rho}_{b} \hat{\rho}_{c} \hat{f}^{\dagger}$ where

$$
\hat{f}=[\hat{a}+\exp (i \gamma) \hat{b}]^{j}[\hat{a}-\exp (i \gamma) \hat{b}]^{k}
$$

After the measurement of the $a$ and $c$ fields, with $l$ and $m$ counts in the detectors, that follows this the (unnormalized) state will be of the form $\hat{g} \hat{f} \hat{\rho}_{a} \hat{\rho}_{b} \hat{\rho}_{c} \hat{f}^{\dagger} \hat{g}^{\dagger}$ where

$$
\hat{g}=\left[\hat{a}+\exp \left(i \gamma_{1}\right) \hat{c}\right]^{l}\left[\hat{a}-\exp \left(i \gamma_{1}\right) \hat{c}\right]^{m}
$$

with $\gamma_{1}$ being determined by path lengths in this measurement. Now, because $\hat{f}$ and $\hat{g}$ commute, this is the same state as $\hat{f} \hat{g} \hat{\rho}_{a} \hat{\rho}_{b} \hat{\rho}_{c} \hat{g}^{\dagger} \hat{f}^{\dagger}$ and so the result of subsequent measurement of the $a$ and $b$ fields will be independent of whether we do the $a$ and $c$ measurement first, then the initial $a$ and $b$ measurement and then the subsequent $a$ and $b$ measurement or whether we do the initial $a$ and $b$ measurement first, then the $a$ and $c$ measurement and then the subsequent $a$ and $b$ measurement. It then follows from our discussion above that the only effect of the intervening $a$ and $c$ measurement will be the negligible depletion effect.

The third question of interest is whether or not there will be a non-random phase relationship between the fields of cavities $b$ and $c$ after we have made a balanced homodyne measurement of the fields from cavities $a$ and $b$ and then a measurement of the fields from $a$ and $c$. After $l$ and $m$ counts in the detectors for the second measurement the state of the 
three cavity fields will be, as above, of the form

$$
\hat{\rho}_{g} \propto \hat{g} \hat{f}_{\rho_{a}} \hat{\rho}_{b} \hat{\rho}_{c} \hat{f}^{\dagger} \hat{g}^{\dagger}
$$

We first use the expansion (35) for $\hat{\rho}_{a}$ and then the corresponding expansion for $\hat{\rho}_{b}$. This gives a multiple integral containing a factor $|r(\eta, \Delta)|^{2}$ given by $(37)$ with $\Delta=\varphi+\gamma-\theta$. We can then expand $\hat{\rho}_{c}$ likewise in terms of $|\chi\rangle$, which is an eigenstate of $\hat{c}$ with eigenvalue $\chi$, whose argument is $\zeta$. This gives a corresponding factor $\left|q\left(\eta_{1}, \Delta_{1}\right)\right|^{2}$ where $\Delta_{1}=\zeta+\gamma_{1}-\theta$. The expression corresponding to $(37)$ is

$$
\left|q\left(\eta_{1}, \Delta_{1}\right)\right|^{2}=G\left(\eta_{1}, \Delta_{1}\right)\left(|\alpha|^{2}+|\chi|^{2}\right)^{l+m}
$$

with the relation corresponding to (20) being

$$
G\left(\eta_{1}, \Delta_{1}\right)=\left(1+\eta_{1} \cos \Delta_{1}\right)^{l}\left(1-\eta_{1} \cos \Delta_{1}\right)^{m}
$$

With these expansions, $\hat{\rho}_{g}$ will be given by a multiple integral with a factor

$$
|r(\eta, \Delta)|^{2}\left|q\left(\eta_{1}, \Delta_{1}\right)\right|^{2}|\alpha\rangle|\beta\rangle|\chi\rangle\langle\chi|\langle\beta|\langle\alpha|
$$

appearing in the integrand.

For large photocount numbers, as we are assuming, $\mid\left(\left.r(\eta, \Delta)\right|^{2}\right.$ and $\left|q\left(\eta_{1}, \Delta_{1}\right)\right|^{2} \mid$ are sharply peaked functions of $\Delta$ and $\Delta_{1}$ with maxima at $\Delta= \pm \Delta_{0}$ and $\Delta_{1}= \pm \Delta_{01}$ respectively. Thus when integrating with respect to $\varphi$ the dominant contributions will be for values $\varphi= \pm \Delta_{0}-\gamma+\theta$. Thus we can write $\hat{\rho}_{g}$ as the sum of two equally weighted multiple integrals, one containing the coherent state ||$\beta\left|\exp \left[i\left(\Delta_{0}-\gamma+\theta\right)\right]\right\rangle$ and the other containing the coherent state ||$\beta\left|\exp \left[i\left(-\Delta_{0}-\gamma+\theta\right)\right]\right\rangle$. Similarly when integrating with respect to $\zeta$ the dominant contributions will be for values $\zeta= \pm \Delta_{01}-\gamma_{1}+\theta$. This allows us to split $\hat{\rho}_{g}$ into four equally weighted terms,

$$
\hat{\rho}_{g}=\frac{1}{4} \hat{\rho}_{g 1}+\frac{1}{4} \hat{\rho}_{g 2}+\frac{1}{4} \hat{\rho}_{g 3}+\frac{1}{4} \hat{\rho}_{g 4}
$$

where the four multiple integrals for $\hat{\rho}_{g 1}, \hat{\rho}_{g 2}, \hat{\rho}_{g 3}$ and $\hat{\rho}_{g 4}$ contain the following product states respectively:

$$
\begin{gathered}
|\alpha\rangle|| \beta\left|\exp \left[i\left(\Delta_{0}-\gamma+\theta\right)\right]\right\rangle|| \chi\left|\exp \left[i\left(\Delta_{01}-\gamma_{1}+\theta\right)\right]\right\rangle \\
|\alpha\rangle|| \beta\left|\exp \left[i\left(\Delta_{0}-\gamma+\theta\right)\right]\right\rangle|| \chi\left|\exp \left[i\left(-\Delta_{01}-\gamma_{1}+\theta\right)\right]\right\rangle
\end{gathered}
$$




$$
\begin{array}{r}
|\alpha\rangle|| \beta\left|\exp \left[i\left(-\Delta_{0}-\gamma+\theta\right)\right]\right\rangle|| \chi\left|\exp \left[i\left(\Delta_{01}-\gamma_{1}+\theta\right)\right]\right\rangle \\
|\alpha\rangle|| \beta\left|\exp \left[i\left(-\Delta_{0}-\gamma+\theta\right)\right]\right\rangle|| \chi\left|\exp \left[i\left(-\Delta_{01}-\gamma_{1}+\theta\right)\right]\right\rangle
\end{array}
$$

Using the strong field approximation (28), we find that each of these is an approximate eigenstate of $\exp \left[i\left(\hat{\phi}_{b}-\hat{\phi}_{c}\right]\right.$. For example the approximate eigenvalue of (59) is $\exp \left[i\left(\Delta_{0}-\right.\right.$ $\left.\left.\Delta_{01}-\gamma+\gamma_{1}\right)\right]$. The value of $\Delta_{0}$ is given by (21) and (22). For narrow number state distributions, as we are assuming here, dominant contributions to the integrations over $|\alpha|$ and $|\beta|$ will be from values of $|\alpha|^{2}$ and $|\beta|^{2}$ near the respective mean photon numbers. This means we can replace $\Delta_{0}$ by $\bar{\Delta}_{0}$ given by (50) and (51). We can similarly replace $\Delta_{01}$ by $\bar{\Delta}_{01}$ which is similar to (50) and (51) but with $\bar{n}_{c}$ in place of $\bar{n}_{b}$. We then obtain

$$
\exp \left[i\left(\hat{\phi}_{b}-\hat{\phi}_{c}\right] \hat{\rho}_{g 1} \approx \exp \left[i\left(\bar{\Delta}_{0}-\bar{\Delta}_{01}-\gamma+\gamma_{1}\right)\right] \hat{\rho}_{g 1}\right.
$$

showing that $\hat{\rho}_{g 1}$ is a state of well-defined phase difference $\bar{\Delta}_{0}-\bar{\Delta}_{01}-\gamma+\gamma_{1}$. Similarly $\hat{\rho}_{g 2}$, $\hat{\rho}_{g 3}$ and $\hat{\rho}_{g 4}$ are states with well-defined phase differences $\bar{\Delta}_{0}+\bar{\Delta}_{01}-\gamma+\gamma_{1},-\bar{\Delta}_{0}-\bar{\Delta}_{01}-\gamma+\gamma_{1}$ and $-\bar{\Delta}_{0}+\bar{\Delta}_{01}-\gamma+\gamma_{1}$.

The presence of four possible phase differences is due to that fact that the balanced homodyne measurements induces two possible phase differences between the $a$ and $b$ cavities and also two between the $a$ and $c$ cavities. If we wished to establish a single phase difference between the $b$ and $c$ cavities we could change $\gamma$ to $\gamma-\pi / 2$ halfway through the homodyning of the $a$ and $b$ fields as described earlier and change $\gamma_{1}$ to $\gamma_{1}-\pi / 2$ halfway through the homodyning of the $a$ and $c$ fields.

The above arguments can be extended to more cavities, enabling the construction of a laboratory in the sense used by Aharonov and Susskind [28], which includes an arbitrary numbers of cavities that serve as frames of reference. One cavity may be chosen arbitrarily as the zero of phase. By means of a homodyne experiment we can prepare a cavity field in a state that can be treated as a superposition of number states with a definite phase relative to that origin. This phase can be checked at any other cavity by an interference experiment. Consistent results will be obtained within the laboratory in that the phase of the superposition will be independent of the cavity at which it is checked. Thus we can assign definite phases to the intra-cavity fields as long as we work within the confines of the laboratory, even though these fields would have a completely random phase relative to a field external to the laboratory which may have been defined to have, for example, a zero 
phase. This laboratory, of course, would only exist for a time that is short compared to the cavity decay times. In practice an effective Aharonov-Susskind laboratory is usually made from laser beams by beam splitting light from a single master laser. Again, optical coherence in the master laser cavity state is not necessary for this; it is not difficult to show that states obtained even from beam splitting a photon number state are entangled, giving them a non-random phase difference.

Another interesting question is as follows. Suppose we set up an Aharonov-Susskind laboratory with some cavities as described above. What would happen if we then performed a homodyne measurement of one of the cavities with a cavity that had somehow been prepared in a pure coherent state? To be specific, let us assume that a phase difference has been estabished between the fields of cavity $a$ and cavity $b$. We now do a homodyne measurement with cavity $a$ and cavity $c$ but let the state in $c$ be a pure coherent state $|\chi\rangle$ instead of a mixture as we had before. The calculation is similar to finding $\hat{\rho}_{g}$ above, except that this time we do not integrate over $|\chi|$ and $\zeta$. We still find when integrating with respect to $\varphi$ that the dominant contributions will be for values $\varphi= \pm \Delta_{0}-\gamma+\theta$. When integrating with respect to $\theta$ the dominant contributions will be for values $\theta=\zeta \pm \Delta_{01}+\gamma_{1}$. Thus the field of cavity $a$ will have a phase $\zeta \pm \Delta_{01}+\gamma_{1}$ and the field of cavity $b$ will have a phase of $\zeta \pm \Delta_{0}-\gamma \pm \Delta_{01}+\gamma_{1}$. If during the measurements we change $\gamma$ and $\gamma_{1}$ appropriately we obtain a single value for the phase in each cavity. This method of homodyning the field from just one cavity with an external coherent state allows us to give a definite phase to all the cavities in the Aharonov-Susskind laboratory. Similarly we can establish definite phase differences between the cavity fields of two different Aharonov-Susskind laboratories. Corresponding arguments apply to laboratories made from splitting laser beams.

\section{PHASE COHERENCE OF A SINGLE LASER BEAM}

We mentioned earlier that successive homodyne measurements indicate phase coherence between sequential packets of light emitted by a laser cavity even if there is no initial optical coherence of the light within the cavity. The case we first examined involved a Poisson distribution of photon number states, which might be interpreted in terms of a mixture of coherent states of known amplitude but unknown phase, allowing the initial random phase to be interpreted in terms of our ignorance of the actual phase. If we adopt 
this interpretation, the measurement then has the essentially retrodictive effect of simply reducing this ignorance. We then said that for the more general case, where the distribution is not necessarily Poissonian, the external field can be entangled with the internal field which gives a means by which a measurement of the external field can physically alter the internal field. However the fact that the internal state can still be written as a mixture (15) of partial phase states might indicate that the change might still be at least mainly due to information being obtained by the measurement about which partial phase states are actually there. It is worth therefore looking at the more extreme but reasonably simple case of cavity a prepared in a pure photon number state $|N\rangle_{a}$, where $N$ is reasonably large, and cavity $b$ being in a pure coherent state $|\beta\rangle$. In this case each partial phase state (16) is the number state itself and so obtaining information about which of these partial phase states can be present initially will not help. For this case we find eventually that for $N>>j+k$ expression (10) for $\hat{\rho}_{f}$ after homodyning can be written approximately, in place of (38) and $(39)$, as

$$
\begin{gathered}
\hat{\rho}_{f} \propto|\beta\rangle\langle\beta|\left[\left|N^{1 / 2} \exp \left(i \theta_{+}\right)\right\rangle\left\langle N^{1 / 2} \exp \left(i \theta_{+}\right)\right|\right. \\
\left.+\left|N^{1 / 2} \exp \left(i \theta_{-}\right)\right\rangle\left\langle N^{1 / 2} \exp \left(i \theta_{-}\right)\right|\right]
\end{gathered}
$$

where the kets and bras of the second factor represent coherent states with mean phases $\theta_{+}=\varphi+\gamma+\Delta_{0}$ and $\theta_{-}=\varphi+\gamma-\Delta_{0}$. If we then change $\gamma$ by $\pi / 2$ and repeat the experiment we can eliminate one of the states of the superposition and obtain a final state that is approximately the product of two coherent states. We note that in this case the measurements have left the cavity $b$ state as $|\beta\rangle$ with phase $\varphi$ unaltered but have physically converted the original pure photon number in cavity $a$ into an approximate coherent state whose phase depends on the outcome of the measurement. Specifically, if the second state in (64) is eliminated for example, the phase of the final state in cavity $a$ is

$$
\theta_{+}=\varphi+\gamma+\cos ^{-1}\left[\frac{\left(N+|\beta|^{2}\right)(j-k)}{2 N^{1 / 2}|\beta|(j+k)}\right] \text {. }
$$

Subsequent light emitted by cavity $a$ would have this phase.

The above shows that if we regard the cavity $a$ as emitting a sequence of packets of light each with the same duration [9], then there would be a correlation between the phase indicated by a homodyne measurement of the first packet and the phase indicated by a measurement of the second packet, even if the initial state of the cavity were a pure photon 
number state. The mechanism producing this correlation is the induced change in the internal field arising from the measurement of the first packet. An interesting question is whether there would be an intrinsic phase coherence, that is a non-random phase difference, between the two packets in the absence of a measurement when the cavity is in a pure number state. We can model this by allowing cavity $a$, initially in state $|N\rangle_{a}$, to interact for a time $T$ with the external mode associated with the first packet, initially in the vacuum state $|0\rangle_{1}$, via an energy-conserving interaction with Hamiltonian $\hat{H}_{1}$ given by $\epsilon \hat{a} \hat{a}_{1}^{\dagger}+$ h.c. similar to what we have used before. We then remove this packet and allow the cavity to interact for time $T$ with the second packet mode via a similar Hamiltonian $\hat{H}_{2}$. The resulting combined state of the cavity and the first and second packets is

$$
\exp \left(-i \hat{H}_{2} T\right) \exp \left(-i \hat{H}_{1} T\right)|N\rangle_{a}|0\rangle_{1}|0\rangle_{2}
$$

The unitary evolution covers the whole interaction time because there are no measurements to terminate it. Expanding (66) to first order we find the state is

$$
|N\rangle_{a}|0\rangle_{1}|0\rangle_{2}-i \epsilon T|N-1\rangle_{a}\left(|1\rangle_{1}|0\rangle_{2}+|0\rangle_{1}|1\rangle_{2}\right)
$$

The first term alone would give a random phase difference between the two packets, that is, a phase difference variance of $\pi^{2} / 3$. The state $|1\rangle_{1}|0\rangle_{2}+|0\rangle_{1}|1\rangle_{2}$ has a mean phase difference of zero with a substantially reduced variance of $\left(\pi^{2} / 3\right)-2[14,18]$. This can be shown from the general expression for the phase difference distribution in Ref. [29]. States in the higher order terms all have zero mean phase difference and reducing variances, leading to an overall phase coherence of the packets of laser light with each other. It is quite clear from the above that the phase coherence in this case does not arise from optical coherences in the prepared internal cavity state. A simple mechanism for its origin can be described as follows. Suppose, to be definite, that we know by some means that, after the time $2 T$ taken to emit two successive packets of light, the cavity has lost one photon. This photon has an equal amplitude of being found in either of the two packets and not in the other, which gives rise to the entangled state $|1\rangle_{1}|0\rangle_{2}+|0\rangle_{1}|1\rangle_{2}$ which has a non-random phase difference. If we wish to look at intrinsic phase coherence of the light beam for longer interaction times $T$ and for more general internal states, the simplest method is to expand the internal density operator in terms of coherent states $|\alpha\rangle$ as in (35). The above unitary transformation then converts $|\alpha\rangle|0\rangle_{1}|0\rangle_{2}$ to $\left|\alpha^{\prime}\right\rangle\left|\alpha_{1}\right\rangle_{1}\left|\alpha_{2}\right\rangle_{2}$ where $\alpha_{1}$ and $\alpha_{2}$ have the same argument. Thus the 
mean phase difference between the two external states is zero with a small phase difference variance for reasonable intensities. This zero phase difference between the packets survives the final integration over $\alpha$ resulting in the intrinsic phase coherence of the beam. As this argument is applicable for any internal state, intrinsic phase coherence can be seen to be more a consequence of the nature of the coupling between the internal and external modes than of the internal state itself (see also Ref. [16]).

\section{CONCLUSION}

We have examined the interference effects in the light from two different laser cavities, building on a physical explanation offered by Pfleegor and Mandel [6] many years ago. The paper extends more recent work $[8,17]$ in that the states of the light inside the cavities can be quite general, and need not be similar, as long as these states are intense enough for us to neglect depletion effects during the total detection time, which must be shorter than the laser pumping time. Even when the initial cavity states contain no optical coherences, the interference effects are quite pronounced, confirming the view of Pfleegor and Mandel [6] who cautioned against associating their observed interference effect too closely with particular states of the two laser beams. In their opinion it is better to associate the interference with the detection process itself and they speculated that similar results should be obtainable with a wide range of possible states of the two fields being superposed.

In support of the above suggestion of Pfleegor and Mandel, we find that, in general, a balanced homodyne measurement on light leaking from two cavities will create a phase difference between the internal states of the cavities, which in turn will produce a signal in a subsequent homodyne measurement that is consistent with the initial signal. If the initial cavity states have reasonably narrow number state distributions then, provided sufficient photocounts are recorded in the original measurement, the subsequent signal can mimic that expected from two classical states with well-defined amplitudes and phases.

By means of an extension to a third cavity we have shown in detail that it is possible to construct a laboratory in the sense used by Aharonov and Susskind [28], which includes an arbitrary numbers of cavities that serve as frames of reference. As long as we restrict ourselves to work within the confines this laboratory we can assign phases to the intracavity fields, which then have phase coherence relative to each other, that is, well-defined 
phase differences. By homodyning the field from just one cavity of the Aharonov-Susskind laboratory with an external coherent state we can give a definite phase to the field of every cavity in the laboratory. By this we mean a definite phase difference between these cavity fields and coherent states in the external world, or at least in the larger Aharonov-Susskind laboratory in which we perhaps live.

Phase coherence can also be associated with packets of light in a single laser beam. If the beam is considered to be divided into successive packets of light then these successive packets will have well-defined phase differences, giving the beam what we might term an intrinsic phase coherence. This occurs even when the intra-cavity state is initially in a pure photon number state. The fact that such phase coherence can arise independently of the pumping mechanism of the laser and of whether or not the internal cavity state has optical coherences indicates that it is more a consequence of the nature of the coupling between the internal and external cavity modes.

Finally, as stated earlier, it is not the intention of this paper to enter the controversy over whether or not a typical laboratory laser really produces light in a coherent state. There is a widely held view that for all practical purposes a laser does produce a coherent state as evidenced by the fact that this assumption has led to successful predictions of experimental results for many years. Furthermore the density operator for a mixture of coherent states of known amplitude but unknown mean phase is identical to that for a mixture of number states with a Poisson probability distribution, and it is only the density operator itself, not its interpretation, that determines the statistics of future measurement outcomes made on the laser beam alone. This raises two questions. The first is a practical question: if coherent states, which are relatively easy to use, are sufficient to calculate experimental results, why consider other possibilities? The second question concerns the nature of physical reality: does it mean anything to say that a laser does or does not really produce a coherent state if such an assertion cannot be proven by experiment? In relation to the second question it is very unlikely, whatever the reality of the situation, that without experimental evidence the prevailing view that a typical laboratory laser does produce a coherent state will change. Is such evidence possible, even in principle, given what we have said above about measurements made on the laser beam? Certainly the possibility of obtaining such evidence does not seem likely within the confines of the Aharonov-Susskind laboratory built by beam splitting the light from a master laser. Also doing an experiment to measure the phase of the master 
laser relative to an external source does not seem promising as the measurement itself can create a phase difference. If such evidence is obtainable, it would probably need to be associated with a property of a coherent state other than its phase. A possibility might be as follows. When coherent states are produced by a source, these states tend not to be entangled with the source, so evidence of entanglement with the source might be evidence against the production of a coherent state. In this case the atoms in the laser cavity are the source of the internal field. Perhaps evidence of some entanglement between the light emitted by the atoms through the sides of the cavity and the light in the beam might be useful. We shall not, however, speculate further on this. The first question above as to why we should consider other possibilities is easier to answer. Such a consideration leads to a study of more interesting peculiarly quantum effects than the simple use of a coherent state that tends not to be entangled with anything. For example, we have seen that an intrinsic

phase coherence in a laser beam can arise even for an internal cavity field such as pure number state. The far-sighted view of Pfleegor and Mandel [6] that it is better to associate the interference with the detection process itself rather than with particular states of the laser beams is an ideal starting point for this study.

\section{Acknowledgments}

I thank the Australian Research Council and the Queensland Government for funding.

[1] P. A. M. Dirac, The Principles of Quantum Mechanics (Clarendon, Oxford, 1958).

[2] A. T. Forester, R. A. Gudmundsen, and P. O. Johnson, Phys. Rev. 99, 1691 (1955).

[3] R. Hanbury Brown and R. Q. Twiss, Nature 177, 27 (1956).

[4] G. Magyar and L. Mandel, Nature 198, 255 (1963).

[5] R. L. Pfleeger and L. Mandel, Phys. Lett. A 24, 766 (1967).

[6] R. L. Pfleeger and L. Mandel, Phys. Rev. 159, 1084 (1967).

[7] S. J. Kuo, D. T. Smithey, and M. G. Raymer, Phys. Rev. A 43, 4083 (1991).

[8] K. Mølmer, Phys. Rev. A 55, 3195 (1997); K. Mølmer, J. Mod. Optics 44, 1937 (1997).

[9] S. J. van Enk and C. A. Fuchs, Phys. Rev. Lett. 88, 027902 (2002). 
[10] J. Gea-Banacloche, Phys. Rev. A 58, 4244 (1998).

[11] K. Mølmer, Phys. Rev. A 58, 4247 (1998).

[12] S. D. Bartlett, T. Rudolph, and R. W. Spekkens, Int. J. Quant. Information 4, 17 (2006); quant-ph/0507214 (2005).

[13] R. Ghosh and L. Mandel, Phys. Rev. Lett. 59, 1903 (1987).

[14] D. T. Pegg, Aust. J. Phys. 46, 77 (1993).

[15] J. Javanainen and S. M. Yoo, Phys. Rev. Lett. 76, 161 (1996).

[16] B. C. Sanders, S. D. Bartlett, T. Rudolph, and P. L. Knight, Phys. Rev. A, 68, 042329 (2003).

[17] H. Cable, P. L. Knight, and T. Rudolph, Phys. Rev. A 71, 042107 (2005).

[18] D. T. Pegg and J. Jeffers, J. Mod. Opt. 52, 1835 (2005).

[19] K. Kraus, States, Effects, and Operations (Springer-Verlag, Berlin, 1983).

[20] S. M. Barnett and P. M. Radmore, Methods in Theoretical Quantum Optics (Oxford University Press, Oxford, 1997).

[21] M. D. Srinivas and E. B. Davies, Optica Acta, 28, 981 (1981).

[22] D. T. Pegg and S. M. Barnett, Europhys. Lett. 6, 483 (1988); S. M. Barnett and D. T. Pegg, J. Mod. Optics 36, 7 (1989); D. T. Pegg and S. M. Barnett, Phys. Rev. A 39, 1665 (1989).

[23] D. J. Rowe, H. de Guise, and B. C. Sanders, J. Math. Phys. 42, 2315 (2001).

[24] S. Watanabe, Rev. Mod. Phys. 27, 179 (1955); Y. Aharonov, P. G. Bergmann, and J. L. Lebowitz, Phys. Rev. 134, B1410 (1964); S. M. Barnett, D. T. Pegg, and J. Jeffers, J. Mod. Optics 47, 1779 (2000); K. L. Pregnell and D. T. Pegg, J. Mod. Optics 51, 1613 (2004) and references therein.

[25] D. T. Pegg, S. M. Barnett, and J. Jeffers, J. Mod. Optics 49, 913 (2002).

[26] P. Kok and S. L. Braunstein, Phys. Rev. A 61, 042304 (2000).

[27] T. Rudolph and B. C. Sanders, Phys. Rev. Lett. 87, 077903 (2001).

[28] Y. Aharonov and L. Susskind, Phys. Rev. 155, 1428 (1967).

[29] D. T. Pegg and S. M. Barnett, J. Mod. Optics 44, 225 (1997). 